\section{Paul K. Feyerabend}

the arts, and makes it possible to speak of styles and preferences in the first, and of progress in the second.

220. Cf. B. Brecht, "Ueber das Zerpfluecken von Gedichten," Uber Lyrik (Frankfurt: Suhrkamp, 1964). In my lectures on the theory of knowledge I usually present and discuss the thesis that finding a new theory for given facts is exactly like finding a new production for a well-known play. For painting see also E. Gombrich, Art and Ilusion (New York: Pantheon, 1960).

221. "The picture of society which we construct we construct for the river-engineers, for the gardeners .... and for the revolutionaries. All of them we invite into our theater, and we ask them not to forget their interest in entertainment when they are with us, for we want to turn over the world to their brains and hearts so that they may change it according to their wishes." Brecht, "Kleines Organon für das Theater," Schriften zum Theater (Frankfurt: Suhrkamp, 1964), p. 20; my italics.

\section{A Picture Theory of Theory Meaning}

The standard view of scientific theories that Herbert Feigl has pictorially set out here is very helpful in some respects. It is a representation of a scientific theory and the role that it plays with respect to the over-all scientific activity in terms of the syntactical calculus of a theory, the observation statements, and the correspondence rules (the coordinating definitions). What has sometimes been characterized as the ideal language conception of the function of a scientific theory and, on the other hand, what has sometimes been characterized as the black box conception of a scientific theory-both stand out in very clear logical relief in terms of this sort of representation. Historically, there are two extremely good cases, one of which would correspond to the ideal language representation and the other to the black box representation.

The ideal language case is the ideal fluid mechanics of Euler and Bernoulli. Fluid mechanics as represented in the post-Newtonian work of these men was a remarkable theory. They saw to it that at the theoretical level the definitions were so sharp and so idealized that the remainder of the algorithm which was generated was very elegant indeed. From the mathematical point of view it is quite a beautiful theory. All that is required is that the subject matter, in this case fluids, be ideal. That is to say, inviscid, irrotational, incompressible, and obeying something called the sine square law. Of course, it turns out that when one makes the algorithm go as beautifully as Euler and Bernoulli did-and to achieve this they had to set out some very clear axioms or definitions by which it could be made a virtual Euclidean instantiation-then a slightly awkward business comes up at the level of the correspondence rules, or the coordinating activities. As it turns out, there are no ideal fluids. In point of fact, the theory doesn't apply to a damned thing, unless one is prepared to bring in lots of additional qualifications at this level. It becomes, then, a sort of general approximation, in terms of which one can have a kind of gestalt for what types of subject matter fluids really are. One must 


\section{N. R. Hanson}

be prepared to muddy up the calculations with the actual values that have to be plugged into the variable terms if one wants to be any good in civil engineering, plumbing, or the designing of boats. If fluid mechanics is going to make any difference to people of that sort, then a great many adjustments have to be effected right at this practical level. In ather words, the objective in the ideal language approach is that certain criteria, formal or at least logical, be met in order that the theory should qualify as being well made. I chose fluid mechanics because it seems to me that this is a historical example of a theory which does meet these criteria up to the hilt.

Contrast the ideal language view with a quite different conception, the black box view. This latter can be represented as the type of theory that is very much concerned with the observed values which one is going to plug into the variable terms of the theory and which coordinate with something that actually constitutes experience. Consider some of the developments of the quantum theory in the late 1930's and certainly in the 1940's. I have in mind now quantum electrodynamics. When that theory concerns itself with weak interactions at high energies, it turns out that certain algorithmic techniques, such as those we owe to thinkers like Dyson and Tomonaga, in particular the technique of renormalization, are from a formal or intra-algorithmic point of view quite objectionable. When one undertakes the technique of renormalization to cope with what are sometimes referred to as the divergencies in the fully developed quantum theory of radiation, it turns out that there are an infinite number of solutions to a given wave equation. One selects, from this infinite set, on terms that are completely extraneous mathematically, a finite number for further examination. Moreover, the renormalization technique, as has been pointed out by Heisenberg, Kallen, Hamilton, and many others, has further disastrous consequences for our understanding of what the quantum theory of radiation is all about. The scattering matrices become non-Hermitian; ghost states, negative probabilities, and all sorts of strange things come up. One has to adopt a fairly tough-minded physicist's attitude toward these. It follows from the fact that the algorithm is so badly designed that one never undertakes to suppose that the argument in favor of the acceptance of a black box theory could be anything other than success. This is one of the strong arguments behind what used to be called the Copenhagen position, to wit, that the thing works pretty well. From the very fact that it's such a mess at the algorithmic level, it seems to fol-
A PICTURE THEORY OF THEORY MEANING

low immediately that the subject matter itself couldn't possibly be like that. Therefore, the whole function of the theory is to provide us with a kind of computational or calculational machine by means of which, when one plugs the values in for the variable terms and turns the handle, the answers come out right. That is the argument in favor of it. Thus, it is clear that both the ideal language representation and the black box representation can be articulated in terms of the standard contrast between the calculus, the observation statement level, and the coordinating definitions or correspondence rules.

This way of putting it does help us to understand precisely what is at stake in some important scientific theories. Sooner or later we would have come to understand quantum theory in terms of a representation like this. Indeed, ideal fluid mechanics has often been characterized in the way in which I have just done it. I want to subscribe with Adolf Grünbaum to the view that the business of hunting for an all or nothing general account of the relationship between theories, their algorithmic structure, correspondence rules, and the observation statements does seem difficult. It's not simply difficult in the sense that it's a great challenge. It seems to me that there certainly are considerations in view of which one might wonder whether this is ever a feasible undertaking.

The language chosen by several of the contributors to this volume concerns correspondence rules, and concerns them in such a way as to suggest that there is a sort of ordering. I don't want this matter to turn simply on an issue in the context of discovery, but it does seem to me that when Professor Hempel refers to the antecedent vocabulary, and when one speaks of finding coordinating definitions for a previously existing theory, there is a commitment here which transcends strictly logical or formal considerations. If one is going to coordinate a certain term in the theory with certain sorts of measurements or observations, there must be a theory which is there and upon which one is going to work. It looks as if the procedure is always a directed series of steps proceeding from having ascertained that the theory is well designed to finding coordinating rules, or activities, or procedures by means of which one succeeds in splicing the algorithms together with experience. A standard example is Euclidean geometry as an algorithm, with coordinating definitions by which one takes a technical term like 'point' or 'straight line' and makes it correspond to something that can be observed, a taut string, or a ray of light, or optically flat glass viewed on edge, or any such thing, and 


\section{N.R. Hanson}

transforms it slowly into a physical theory, i.e., geometrical optics, or physical optics. That seems to be the usual order. I think that most discussions of these matters have rested on an implicit assumption that that is the standard order. While this is helpful in the context where we're concerned with the appropriate representation of theories like ideal fluid mechanics or quantum electrodynamics because of what we can do in terms of this representation, it isn't adequate in all cases. Peter Achinstein has remarked that when he requests a general account of the problem concerning meaning of observation terms or theory, he's always confronted, on the part of Paul Feyerabend and myself, with an example. This is a remarkable thing to complain about. The long tradition of philosophy suffered precisely because anytime one was trying to get some sort of understanding of particular subject matter, the account got too general much too quickly. I am quite prepared to say that a general account is not my objective at all. I would be quite happy if I could take particular cases and get some understanding concerning the relationship in that theory or in that science between the observational terms, the so-called coordinating activities, the theoretical terms, and the theoretical structure itself.

This way of representing the matter, with what I have assumed to be an additional progresșive arrow built into it, might indeed apply very, very well to certain theories. I think that it's totally inadequate for other theories, and the discipline I am going to discuss here has concerned itself with one of these other sorts of theories. If one were to try to press some of the standard analyses of the structure of scientific theories on this particular scientific discipline, the result would be a very distorted and a very confused analysis of exactly what the conceptual structure of this physical theory actually consists in.

Certainly the contrast between the context of discovery and the context of justification has been pointed up many, many times. Let us grant that what I am about to do doesn't turn primarily on matters of learning theory, or experimental psychology, or sociology, or navel contemplation, or pink pancreatic responses, or whether or not I salivate when a bell is rung. This is not the objective. It is honestly my objective to try to avoid that sort of thing. I would like to consider what might be called the context of strategies, the sorts of reasonable considerations in virtue of which a man's argument might be designed one way rather than another. I want to consider the possibility that in the full accounting of
A PICTURE THEORY OF THEORY MEANING

some important scientific disciplines one might find oneself committed to coordinating the observations and the observation statements in a very significant manner long before there is a full-blown theory in the books. Indeed, with respect to the discipline I have in mind, even at this time there isn't such a full-blown theory. This may be said to be simply a factual matter, a matter for the sociologist to consider. Nonetheless, there seem to be important conceptual considerations which follow with respect to our understanding of the actual activity which counts as scientific reasoning, the dealing with perplexing scientific difficulties.

In important cases, formulating a correspondence rule or a coordinating definition is not always representable as finding those terms in the theory which can be coordinated with "terms in experience" or terms in the observation language. A term like 'is coordinated with' refers to a relationship which operates over two terms. It makes perfectly good sense to suppose that this kind of coordinating activity could operate in either direction. The notion of the coordinating activity does not imply that it must always proceed with one end as prior and stable and the other end picking up its semantical content strictly in terms of the first. It might indeed go in the other direction. Please let me say that I have granted that expositions of quantum electrodynamics and other disciplines as well are helped by analyses along the lines of the standard view, but it seems to me that it fails in some cases. All I'm asking for is that philosophers of science take up the examples of the analysis of scientific theory one at a time, and not try to settle the issue all at once.

The discipline with which I am going to concern myself is fluid mechanics, as understood in the last one hundred and sixty-six years. Fluid mechanics, as we know today, had its origins in Book Two of Newton's Principia. Newton's strategy there was simply to reduce the concerns of Book Two to the analyses set out in Book One. In Book One of the Principia a fairly good geometer's or logician's catechism of punctiform mechanics is set out with great power and great elegance. To a considerable extent, Newton was committed to the belief that any problem within the subject of matter and motion which was a genuine problem was ultimately going to be resolvable in terms of the analysis appropriate to punctiform masses and the "forces" acting on them. Consequently, when in Book Two the problem of a very perplexing, turbulent subject matter, namely fluids, came up for analysis, it seemed quite a natural move for Newton to reduce this complex subject matter to a macro-observational 


\section{N. R. Hanson}

manifestation of what at the fine level could be construed simply as ensembles of point masses in relationship to each other and in relationship also to resultant forces and aggregate motions. Therefore, for Newton, the behavior of fluids was ultimately going to be analyzable as if a fluid was like a hail of very, very fine sand. If one had enough information about the complete state descriptions of each of the constituent particles, one could sum over this and end up with a totally intelligible algorithm applicable to fluids. This was the strategy.

In order for Newton to make this particular move, he had had to make certain assumptions about the nature of the fluid itself, in order that the analytical apparatus of Book One should apply to the domain of Book Two. This is simply the way in which all theoretical scientists undertake to make an apparently untractable subject matter fit the analysis to which they are committed at a given moment. The definitions are the point at which one undertakes to link the subject matter with this particular inferential apparatus. I have already mentioned four of Newton's assumptions about fluids. If one is going to make the move that he undertakes in Book Two, fluids have to be treated as inviscid, irrotational, incompressible, unlike any fluids we ever encounter. Furthermore, they must have the property that if one were to take such a fluid with laminar flow impinging on a plate, then the force exerted by the corpuscles in the fluid will be proportional to the density of the fluid. If the fluid were liquid mercury, there would be a much greater value for this variable than for ordinary water or air. It would be proportional also to the area of the particular resisting plate. A postcard is going to have a different deflectional effect than a barn door. Similarly, the velocity of these particles as they impinge on the side of the plate is of the order of $\mathrm{V}^{2}$. Finally, the joker in the Newtonian pack is that the force will vary as the sine squared of the particular angle, $a$. This has come down to us as the infamous sine square law, by virtue of which it follows that birds can't fly.

This type of analysis, as beautifully precisified in the work of Euler and Bernoulli, this fundamental set of convictions, determined for Newton and for the Newtonians precisely what the nature of the deflection of this plate would be as a result of an impact on the underside. This was the same sort of analysis that Leonardo da Vinci applied to the flight of birds when he wrote in the fifteenth century. For him, the entire capacity of a bird to lift itself through this rather evanescent and immaterial medium was what I will call a waterski effect. It is simply a question of

\section{A PICTURE THEORY OF THEORY MEANING}

the degree to which the medium can be compressed on the underside of the bird's wing; that was what sustained the animal. The conception of a force on the underside of a lifting or liftable plane was then analyzed in terms of punctiform interactions by Newton. Let me just give two examples. If the degree to which an object was deflected in that direction were proportional to the sine squared of the angle $a$, the angle of inclination, then a Boeing 320 would actually succeed in getting airborne, given its gross weight of a hundred and sixty tons, if and only if each of its wings were the size of 2.3 football fields. A hummingbird would need both of his wings, given the nature of his sustentation, with a wing plan about the size of a table. Now, what follows from this?

The price that one pays for making this algorithm so beautiful, as it was in the Newtonian framework, is simply that it applies to absolutely nothing. This is the case with ideal fluid mechanics. In point of fact, those who in the middle of the nineteenth century and later were concerned with practical problems of fluid mechanics discovered that they could no longer appeal to physics, theoretical or experimental, for any help whatever.

Paul Feyerabend and I have been previously linked together as apparently being in agreement at Pittsburgh in 1965 on the fact that the Wright brothers didn't know what an airplane was. That was not the point. The point was that the Wright brothers, in a very serious sense, didn't know what flight was. Understanding precisely what it is that takes place when a winged object, whether animate or inanimate, gets itself up into this rather thin medium was not something that was possible before 1908 . This is just a calendrical fact. Our understanding of what flight consists in has its roots in the work of Lanchester in 1908 on aerodynamics. Whatever made the achievement of practical flight possible was quite independent of anything that could be offered at this particular time by the theory of fluid mechanics. Let me make one slight digression in order to develop a theoretical point which is important for me at this moment. Long before 1908, Stringfellow, Henson, Horatio Phillips, and others undertook to make measurements with primitive wind tunnels on airfoil sections. They did this in a true Keplerian manner with columns and columns of figures concerning the degree of curvature of the plane itself, the velocity, the area, the density of the fluid in question, the planform of the wing, and the angle of inclination. All of these things were set out and made tabloid in the way in which Boyle's data in 1661 would have had to have 


\section{N. R. Hanson}

been presented before the actual generalization which goes under his name was really formulated. One had here a direct encounter at the experiential level; the observation statements were such that they provided these men with the capacity to set out charts of data points.

Suppose that we digress now from airfoil sections to talk about the internal combustion engine. It will very often be useful for the engineer to represent many parameters on precisely the same chart. He might at one time, for example, want to plot the relative brake mean effective pressure delivered by the airscrew, with a given r.p.m., a given mixture setting, and so forth. He can plot other curves as they intersect with it, concerning things like altitude and angles of tack. From the structural relationship between the slopes of these lines, one can get a very acute and intimate understanding of the dynamical processes which operate inside the engine. There is something very important about the relationship of these sloping lines to the processes that one is actually going to find in the subject matter. One knows what it would be like to make a mistake in any one of these data-point representations. There is a mistake when this representation of the structure, concerning this particular parameter, is not in accord with what one actually observes in the complex subject matter. Consequently, it makes sense to say of a representation of data points of this kind that it is veridical. In other words, it is informative concerning the dynamics of the subject matter. In some important sense the structure of this representation is identical with the structure of the dynamical subject matter, or there has got to be a very intimate correlation at the very least.

For example, what one learns is that as time goes on, the relative viscosity of the lubricant will change one way or another, or as the r.p.m. is increased, the relative inefficiency of the valve clearances might make itself more evident. All these things are dynamical influxes and effluxes which can be plotted in terms of data points on this chart. In a very real sense these give one the only insight that matters into what the engine really is-that is, a structural insight. If one were to go to the Pratt and Whitney or the Allison factories and look at a long array of power plants, all of the same design, say the R2800-30W engine, which designates a series of specific performance expectations, it would be idle to distinguish any two of these on the basis that one was rusty and the other was polished, or that one had 'Kilroy' marked on the side, and the other was quite nascent. The important distinction that one makes

\section{A PICTURE THEORY OF THEORY MEANING}

is that an engine either does measure up to the fundamental design strategy that was built into the thing, or fails altogether in having the structure which could be represented in this form. If it fails altogether in having the structure, quality control might reject it as not being an adequate example of the R2800-30W engine. But what makes any two or any twenty of these engines the same engine, and that is the way they will be sold, is that they conform to the specifications set out on a chart of this kind. If there were a chart which recorded the data points of a given engine, and if there were a corresponding chart which plotted the characteristics which the designer had in mind, these lines would be more or less congruent, within some degree of error. In some sense of 'structure,' the data-point representation and the subject matter itself have the same structure. When one has a line sloping in X-Y space, there will be some corresponding algebraic expression which will do in the A, $\mathrm{B}, \mathrm{C}$ variable space precisely what the line in the X-Y space does without any loss of power whatsoever.

The distinction to be drawn between the geometry of a data representation and the corresponding algebraic representation is not a distinction which matters at all, from a logical point of view. If there were an argument in virtue of which one could say that the subject matter and the data-point representation had the same structure, then this point must carry over for the algebraic rendition of precisely the same information. One would then have, with respect to any particular subject matter, a large cluster of expressions, all algebraic in form, which do for one's understanding of the subject matter what the data-point graph did.

According to the general theory of functions, given any two algebraic expressions it will always be possible to find some algebraic expression from which both of them can be generated, within certain boundary con ditions. At this stage, the operation is largely one of mathematical creativity. That is to say, one will undertake to find fewer and fewer higher order algebraic commitments from the statement of which whole series of lower order algebraic commitments can be shown to follow deductively. These lower order statements will, as I stressed before, in some very important sense, have the same structure as the subject matter. So far we are still moving in the "up" direction with respect to our diagram of the standard view of scientific theories. The great question comes when the individual moves to higher and higher levels of abstraction, which it is his objective to achieve. (Is it the case that what one could have 


\section{N. R. Hanson}

said about the structural relation of charts to engines is the same as what could be said about the relation of high-level theory to engines? If this is the case, then we might conclude that the decisions made at the level of coordinating activity determine the theory.)

My point in saying, as I did at Pittsburgh, that the Wright brothers didn't understand what flight was, at which moment Paul Feyerabend dramatically agreed, was simply that the nature of the series of experimental approximations, the trial and error that was undertaken until 1903, in no way gave anyone any understanding of precisely what the nature of flight was. There were still many attempts to deal with the problem of flight in terms of underside pressure on the wing. It was in 1908, after the practical achievement of flight, that Lanchester came forward with the essential idea.

Newton himself very clearly described an effect which follows the placing of a cylindrical rod transversely in a flowing fluid. Newton noticed that if the rod is given a sense of rotation, and if the flow lines are laminar and impinge in the appropriate way, this cylindrical rod will be deflected. This is the Magnus effect which was experimentally well known by the nineteenth century. This is the only thing which could really account for the remarkable fact that, Newtonian fluid mechanics to the contrary, birds do fly, and the Wright brothers got off the ground. The theory of Lanchester was the following. Consider any one of those shapes, which had been shown to be more effective by the experimental work of Wendham and Horatio Phillips. It was not possible to understand fully what this deflection consisted in, in terms of the waterski effect, or the underside pressure. The actual effect always seemed to be very much greater than what could be calculated in terms of the underside effect. But Lanchester, since he knew about the Magnus effect, argued that it was one of the fundamental functions of a shape of this sort to initiate a movement of this kind in the fluid through which the shape was moving. In other words, it was the function of this shape to set up a circulation around the wing. This is the beginning of what we still call the circulation theory of airfoil lift, or the lifting line. To explain this fully requires a considerable amount of boundary-layer theory, which I can't go into here. But the nature of this interaction of matter molecules on the surface of the cylindrical rod and immediately adjacent to it in the fluid is very important. It required work in the 1920's by Prandtl to explicate it fully. If one can suppose that the wing-air interaction generates something like the Magnus effect, then

\section{A PICTURE THEORY OF THEORY MEANING}

many other things follow immediately, such things as coming to understand how the Bernoulli effect gets into the picture. How one builds up the starting vortex of Prandtl and von Karman to explain flight follows immediately from Lanchester's fundamental conception. The marriage of what might be called the physicist's conception of a slightly generalized vortex theory with the actual problem of flight was brought about by Lanchester's fundamental conception. This union has controlled the development of theoretical aerodynamics to the present. At the moment we are a long way from having anything like a well-designed Euclidean algorithm, in any sense. Nonetheless, if there's any motion here, it's in the upward direction. It seems to me that this is not simply something to be identified as lying strictly inside the context of discovery. There are conceptual implications of this for the understanding of how it is that in some sciences the coordinating definitions or the correspondence rules may be related to experience in ways slightly different from what we find in other sciences. 